\title{
Biologische Konsequenzen schwefelsäure- und eisensulfathaltiger Industrieabwässer. Mortalität junger Gobius pictus und Solea solea (Pisces)
}

\author{
Otto Kinne und Karl-Heinz Schumann
}

Biologische Anstalt Helgoland, Meeresstation, Helgoland

\begin{abstract}
Biological consequences of sulphuric acid and iron sulphate-containing industry wastes. Mortality of young, Gobius picius and Solea solea (Pisces). Industrial waste products containing primarily $\mathrm{H}_{2} \mathrm{SO}_{4}$ and $\mathrm{FeSO}_{4}$ (for details consult Table 1) are scheduled to be discharged in quantities of some 1,200 tons per day into a North Sea area beginning 11.5 nautical miles north-west of Helgoland. Discharging is announced to start in early 1969. In view of these plans, the Biologische Anstalt Helgoland has initiated a research programme comprising studies in the field, in large sea-water containers and in artificial eco-systems, as well as mortality and capacity tests of animal, plant and micro-organism representatives of the discharge area. The present study deals with mortality tests conducted on fishes: fry of Gobius pictus (total length: $20 \pm 2 \mathrm{~mm}$ ) and 6-day old larvae of Solea solea (total length: $4.1 \pm 0.2 \mathrm{~mm}$ ). Mortality of G. pictus is, at $18.5^{\circ} \mathrm{C}$, significantly increased down to waste product dilutions (with sea-water of $32 \% \mathrm{~S}$ ) of 1:8000. Our experiments reveal increased mortality also in 1:12000, 1:16000, 1:24000 and 1:32000 (Abb. 2, 3); further investigations are required in order to evaluate the statistical significance of the negative biological consequences suffered in these rather diluted test media. The 50\% lethal dose $\left(L_{50}\right)$ after 24 hours lies near 1:6000. Sublethal consequences (marked temporary increase in frequency and fluctuations of operculum movements) occur down to dilutions of 1:32000 (Abb. 4, 5). S. solea larvae show, at $20^{\circ} \mathrm{C}$, increased mortalities down to waste product dilutions of 1:12500 and 1:15000; the observed mortality increases in 1:17500 and 1:20000 need support through further experiments based on larger numbers of test fish. The $50 \%$ lethal dose ( $\left.L D_{50}\right)$ after 24 hours lies near 1:15000. Thus the $S$. solea larvae are more sensitive to the industrial wastes in question than are the young of $G$. pictus. It is planned to continue and to deepen these studies through further and more detailed experiments. On the whole, our results are quite comparable to those obtained earlier by KINNE \& RosenTHAL (1967) on eggs and larvae of the herring Clupea barengus $\left(8.0^{\circ} \mathrm{C} ; 16.5 \% \mathrm{~S}\right)$.
\end{abstract}

\section{EINLEITUNG}

Ober die biologischen Konsequenzen schwefelsäure- und eisensulfathaltiger Industrieabwässer ist wenig bekannt. Angesichts der für Anfang 1969 in Aussicht genommenen Verklappung von etwa 1200 Tonnen solcher Abwässer pro Tag in einem Meeresgebiet, das 11,5 Seemeilen nordwestlich von Helgoland beginnt, hat die Biologische Anstalt Helgoland mit einer Reihe von Untersuchungen begonnen. Sie haben zum Ziel, einen Beitrag zu leisten zur Beurteilung der zu erwartenden Auswirkungen 
derartiger Maßnahmen auf die marine Fauna und Flora im zukünftigen Verklappungsgebiet. Diese Untersuchungen werden durch das Bundesernährungsministerium und die Deutsche Forschungsgemeinschaft unterstützt.

Das Untersuchungsprogramm der Biologischen Anstalt Helgoland umfaßt Arbeiten in See, großen Seewasserbehältern und künstlichen Okosystemen sowie Mortalitäts- und Leistungstests an Einzelarten im Laboratorium. Bei den Arbeiten in See soll zum Teil in Zusammenarbeit mit dem Deutschen Hydrographischen Institut, der Bundesforschungsanstalt für Fischerei und dem Institut für Meeresforschung in Bremerhaven - zunächst eine eingehende Aufnahme der gegenwärtigen Fauna und Flora, insbesondere der des Bodens, erfolgen. Die Ergebnisse dieser Untersuchungen in situ werden dann später das frühzeitige Erkennen etwaiger, durch Verklappungsmaßnahmen verursachter, Veränderungen erleichtern. Die ersten experimentell-ökologischen Untersuchungen befassen sich noch primär mit der Ermittlung letal wirkender Dosen auf Repräsentanten der Nordseefauna und -flora unter stark vereinfachten Versuchsbedingungen; ihre Aussagekraft bezüglich der im Meer tatsächlich zu erwartenden Konsequenzen ist dementsprechend begrenzt. Erst die Durchführung der geplanten langfristigen Untersuchungen über subletale Effekte (Beeinflussung der Intensität von Wachstum, Nahrungsumsetzung und Fortpflanzung) kann - zusammen mit Experimenten in vereinfachten Okosystemen und in situ - die erforderlichen Informationen für detaillierte Vorhersagen liefern.

In diesem Beitrag werden Versuchsergebnisse vorgelegt, welche an Jungtieren bzw. Larven der Fische Gobius pictus (Fleckengrundel) und Solea solea (Seezunge) erarbeitet worden sind. Methodik und Kriterien weisen diese Studie als eine erste Groberfassung der Giftigkeit der in Frage stehenden Industrieabwässer unter definierten, vereinfachten Umweltbedingungen aus.

Die Beschaffung einer größeren Anzahl vergleichbarer Versuchstiere (Alter, Größe, Vorgeschichte) war recht schwierig. Die Untersuchungen mußten daher mit relativ wenigen Individuen durchgeführt werden.

\section{MEDIEN}

Die getesteten Industrieabwässer bestehen aus einem Gemisch ${ }^{1}$ von Dünnsäure und Grünsalz im Verhältnis 1:2,75. Nach Angaben der Hersteller enthalten diese beiden Komponenten die in Tabelle 1 aufgeführten Substanzen.

Es ist beabsichtigt, ab Anfang 1969 mit einem Spezialschiff ein Gemisch von etwa 850 bis 880 Tonnen Dünnsäure und etwa 350 Tonnen Grünsalz pro 'Tag in folgendes Meeresgebiet einzubringen: $54^{\circ} 20^{\prime} \mathrm{N}-54^{\circ} 25^{\prime} \mathrm{N}$; $7^{0} 35^{\prime} \mathrm{E}-7^{\circ} 39^{\prime} \mathrm{E}$. Die Temperatur der Abwässer wird vermutlich je nach Jahreszeit zwischen $20^{\circ}$ und $40^{\circ} \mathrm{C}$ schwanken.

Vor den Versuchen wurde das Gemisch, um das Grünsalz aufzulösen, auf $60^{\circ} \mathrm{C}$ erhitzt und mit filtriertem (Filter: Original Seitz-Filterschichten, Sorte K 5, Größe 6) Helgoländer Meerwasser (32 $\pm 1,5 \%$ Salzgehalt) verdünnt ( 1 Teil Gemisch +9 Teile

1 Das Gemisch stellt das Routineendprodukt der Industriefirma dar. Es wurde - ebenso wie die Angaben über seine chemische Zusammensetzung - freundlicherweise von der Titangesellschaft m.b.H., Leverkusen (Bundesrepublik Deutschland), zur Verfügung gestellt. 
Tabelle 1

Bestandteile der Gemischkomponenten Dünnsäure und Grünsalz. Näheres siehe Text

\begin{tabular}{lrllr}
\multicolumn{2}{c}{ Dünnsäure } & \multicolumn{2}{c}{ Grünsalz } \\
$\mathrm{H}_{2} \mathrm{SO}_{4}$ & $18,3 \%$ & $\mathrm{FeSO}_{4}$ & $50,0 \%$ \\
$\mathrm{FeSO}_{4}$ & $12,6 \%$ & $\mathrm{H}_{2} \mathrm{SO}_{4}$ & $0,25 \%$ \\
$\mathrm{MgSO}_{4}$ & $1,9 \%$ & $\mathrm{TiOSO}_{4}$ & $0,4 \%$ \\
$\mathrm{TiOSO}_{4}$ & $1,1 \%$ & $\mathrm{MgSO}_{4}$ & $2,5 \%$ \\
$\mathrm{Al}_{2}\left(\mathrm{SO}_{4}\right)_{3}$ & $0,2 \%$ & $\mathrm{MnSO}_{4}$ & $0,09 \%$ \\
$\mathrm{MnSO}_{4}$ & $0,14 \%$ & $\mathrm{H}_{2} \mathrm{O}$ & $46,76 \%$ \\
$\mathrm{VOSO}_{4}$ & $0,13 \%$ & 2 & $100,00 \%$ \\
$\mathrm{Cr}_{2}\left(\mathrm{SO}_{4}\right)_{3}$ & $0,01 \%$ & & \\
$\mathrm{H}_{2} \mathrm{O}$ & $65,6 \%$ & & \\
\hline$\Sigma$ & $99,98 \%$ & &
\end{tabular}

Meerwasser). Von dieser Sta mmlösung wurde durch Zugabe von filtriertem Helgoländer Meerwasser eine Reihe von Verdiunnungsstufen hergestellt. Sie umfassen den Bereich von 1:2000 bis 1:32000 und werden im folgenden als Testmedien bezeichnet. Bald nach dem Ansetzen der Testmedien entstanden braune, kolloidale Ausflockungen. Es handelt sich hierbei um Eisenhydroxid. Diese Ausflockungen bilden je nach Verdünnungsstufe, Wasserbewegung und Salzgehalt unterschiedlich große Aggregationen, die allmählich absinken und dazu neigen, an der Oberfläche fester Gegenstände verschiedener Beschaffenheit festzukleben.

Als Kontrollmedium diente filtriertes Helgoländer Meerwasser mit einem Salzgehalt von $32 \pm 1,5 \%$.

\section{VERSUCHSERGEBNISSE}

\section{Experimente an Jungtieren von Gobius pictus}

Es wurden Jungfische der Fleckengrundel Gobius pictus MALM 1863 mit Totallängen von $20 \pm 2 \mathrm{~mm}$ untersucht. Sie waren Anfang August vor Helgoland gefangen und vor Versuchsbeginn mehrere Tage in fließendem Meerwasser bei einer Temperatur von $18^{\circ}$ bis $19^{\circ} \mathrm{C}$ gehältert worden. Während der Hälterungszeit wurden die Jungfische mit fein zerteiltem Miesmuschelfleisch (Mytilus edulis) und Larven von Artemia salina gefüttert. Die unter Hälterungsbedingungen verbliebenen Tiere wuchsen normal auf; andere, unter gleichen Bedingungen gehaltene Individuen pflanzten sich sogar fort.

Die Versuche erfolgten in runden, oben offenen Glasschalen mit einem Durchmesser von $230 \mathrm{~mm}$ in Höhe der Wasseroberfläche (Abb. 1a). Die Glasschalen enthielten in $2000 \mathrm{ml}$ Testmedium oder Kontrollmedium jeweils 10 Versuchstiere. Diese wurden mit Hilfe eines weichen Plastiksiebeinsatzes in bestimmten Zeitintervallen in Glasschalen mit frischen Medien umgesetzt. Die Testmedien waren jeweils 10 Minuten vor dem Umsetzen angesetzt worden (Auffüllen der Stammlösungsanteile 
mit Meerwasser auf $2000 \mathrm{ml}$; umrühren mit Glasstab). Während der hier beschriebenen Versuche wurden Test- und Kontrollmedien nicht durchlüftet und die Versuchstiere nicht gefüttert.

Sämtliche Versuche wurden bei Dauerlicht (Glühbirnen; etwa 200 Lux an der Wasseroberfläche in den Versuchsschalen) und einer Wassertemperatur von $18,5 \pm$ $0,5^{\circ} \mathrm{C}$ durchgeführt.

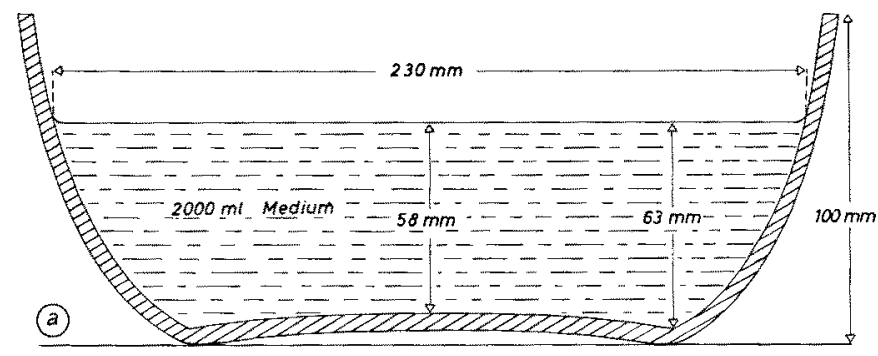

(b)

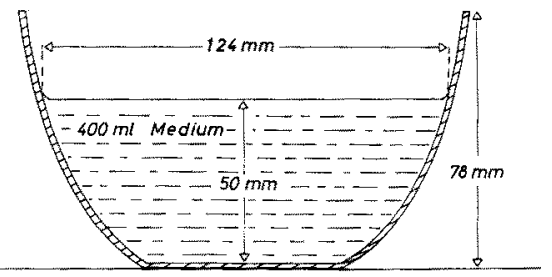

Abb. 1: Die als Versuchsgefäße benutzten runden Glasschalen. a: Versuchsgefäß für Tests an jungen Fleckengrundeln Gobius pictus; $b$ : Versuchsgefäß für Tests an Larven der Seezunge Solea solea

\section{Mortalitätsrate}

Als Kriterium zur Grobbeurteilung der biologischen Konsequenzen der schwefelsäure- und eisensulfathaltigen Industrieabwässer unter den gewählten Versuchsbedingungen diente die Mortalitätsrate (Anzahl der Toten als Funktion der Versuchsdauer). Die Versuchstiere wurden für tot erklärt und entfernt, wenn sie - ohne Atemtätigkeit mit gespreizten Kiemendeckeln und weit geöffnetem Maul - nach Anstoßen mit einem Glasstab keine wahrnehmbaren Bewegungen mehr ausführten. Sie wurden in den ersten 12 Stunden stündlich, danach in 12stündlichen und später in 24stündlichen Intervallen, kontrolliert.

Erste Versuchsreihe. Um einen ersten Überblick über das Ausmaß der Giftigkeit der Testmedien zu gewinnen, wurden junge Gobius pictus in folgende Testmedien überführt: $1: 2000,1: 4000,1: 8000,1: 12000,1: 16000,1: 24000,1: 32000$. Von jedem Testmedium und vom Kontrollmedium wurden 3 Schalen mit je 10 Tieren angesetzt. Die Versuchstiere wurden bei Versuchsbeginn (1. Umsetzen) sowie nach 24 bzw. 48 


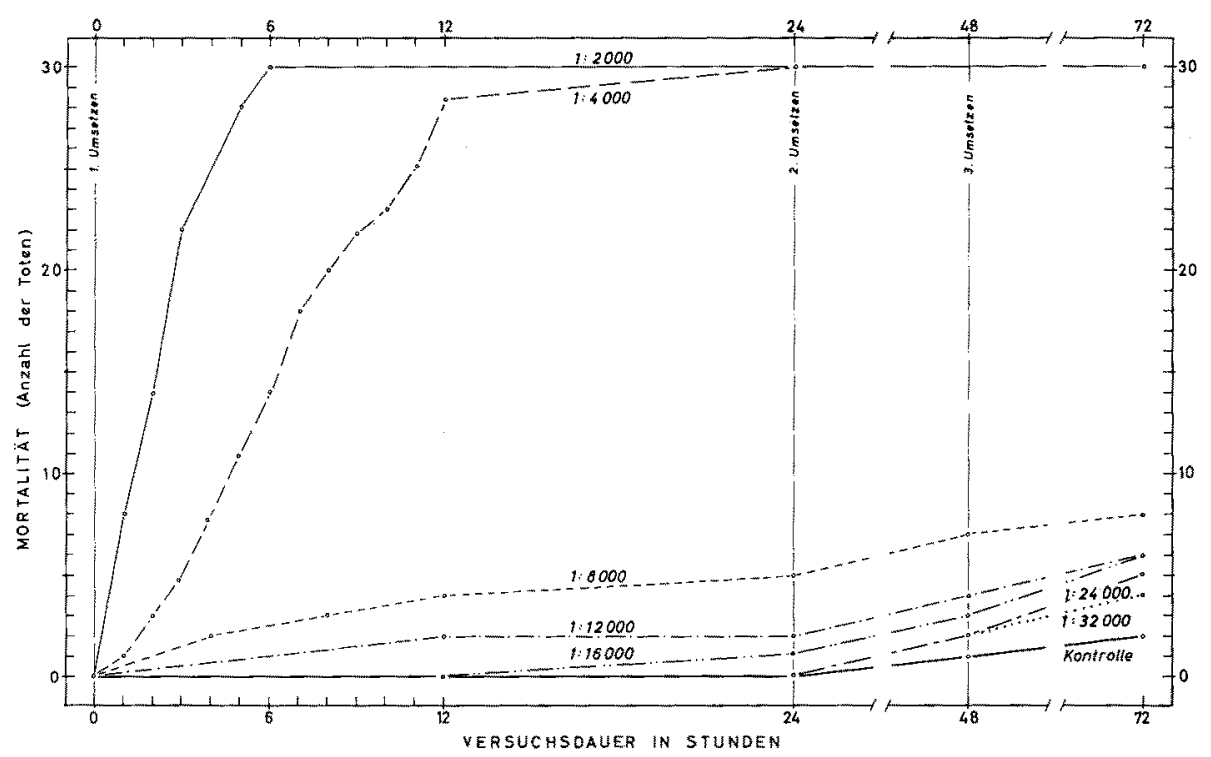

Abb. 2: Mortalität $20 \pm 2 \mathrm{~mm}$ langer Gobius pictus. In 7 Testmedien und im Kontrollmedium wurden jeweils 30 Individuen getestet und die Anzahl der Toten registriert. 1., 2. bzw. 3. Umsetzen: Uberführung der Versuchstiere in die 10 Minuten vorher angesetzten frischen Testmedien bzw. in das 10 Minuten vorher angesetzte frische Kontrollmedium. $\left(18,5^{\circ} \pm 0,5^{\circ} \mathrm{C}\right)$

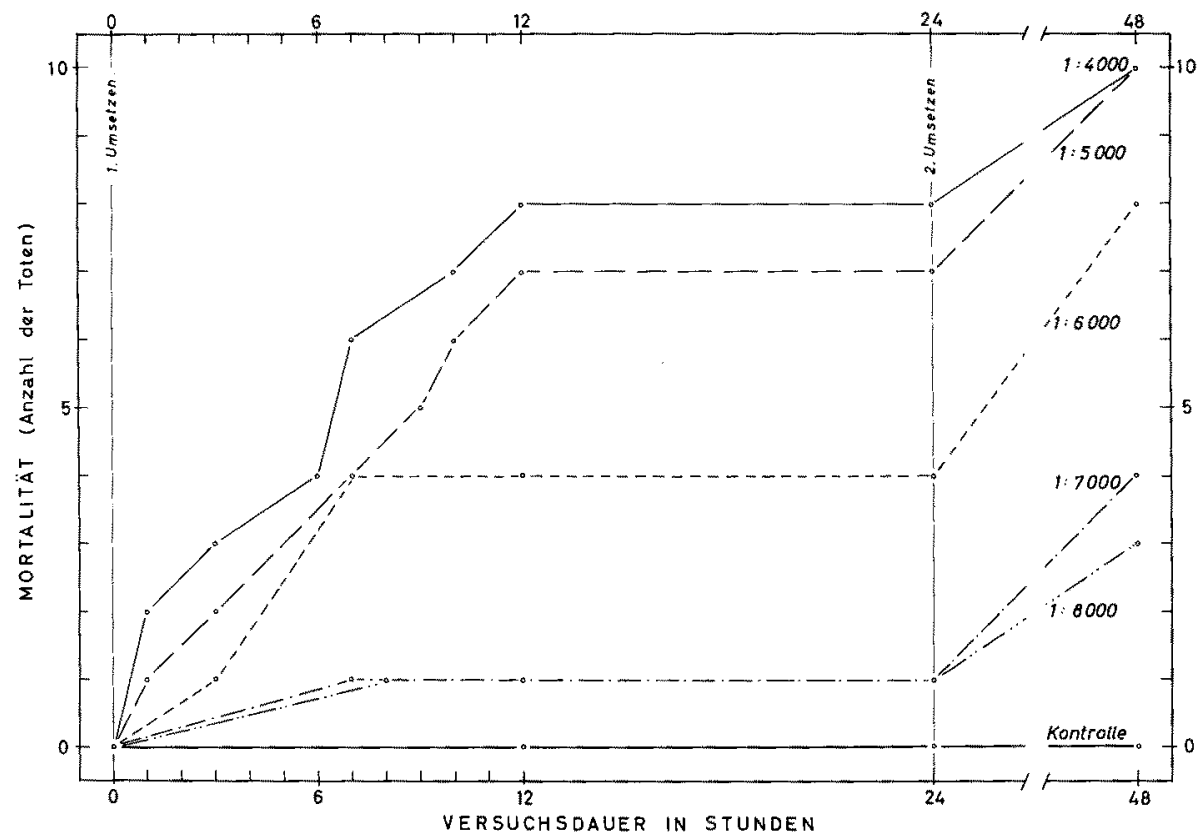

Abb. 3: Mortalität $20 \pm 2 \mathrm{~mm}$ langer Gobius pictus. In 5 Testmedien und im Kontrollmedium wurden jeweils 10 Individuen getestet und die Anzahl der Toten registriert. 1. bzw. 2. Umsetzen: siehe Legende zu Abbildung 1. $\left(18,5^{\circ} \pm 0,5^{\circ} \mathrm{C}\right)$ 
Stunden jeweils gegen 11.00 Uhr morgens in die 10 Minuten vorher angesetzten (siehe oben) Medien umgesetzt.

In 1:2000 und 1:4000 starben sämtliche Versuchstiere innerhalb von 24 Stunden (Abb. 2). In 1:8000 und in den folgenden Verdünnungsstufen war die Sterblichkeit erheblich geringer. Nach Ablauf yon 72 Stunden waren in 1:8000 acht, in 1:12000 und $1: 16000$ je sechs, in 1:24000 fünf, in 1:32000 vier und im Kontrollmedium 2 Individuen gestorben.

$\mathrm{Z}$ weite Versuchsreihe. Die starke Abnahme der Mortalität zwischen 1:4000 und 1:8000 gab Veranlassung zur Durchfuhrung einer zweiten Versuchsreihe mit folgenden Medien: 1:4000, 1:5000, 1:6000, 1:7000, 1:8000, Kontrolle. Für diese Versuche wurden die noch verbliebenen, vergleichbaren Versuchstiere (gleiche Größe und Vorgeschichte) verwandt und in jedem Medium eine Schale mit 10 Versuchstieren angesetzt. Die Versuchstiere wurden bei Testbeginn sowie nach 24 Stunden (jeweils gegen 11.00 Uhr morgens) in die 10 Minuten vorher angesetzten Medien umgesetzt. Wie aus Abbildung 3 hervorgeht, waren in 1:4000 und 1:5000 nach 48 Stunden sämtliche Versuchstiere gestorben; zum gleichen Zeitpunkt waren in 1:6000 acht, in 1:7000 vier und in 1:8000 drei Tiere tot. In der gleichen Zeit starb im Kontrollmedium keines der Versuchstiere.

\section{Kiemendeckelfrequenz}

Die Kiemendeckelfrequenz (pro Minute durchgeführte Kiemendeckelbewegungen) wurde bei Gobius pictus im Verdünnungsbereich 1:4000 bis 1:32000 ermittelt. Hohe Frequenz war meist mit starken Kiemendeckelausschlägen, niedrige Frequenz dagegen mit geringeren Ausschlägen korreliert. Es wurden 2 Versuchsserien durchgeführt.

Erste Versuchsserie. In jedem Testmedium (1:4000, 1:8000 und 1:12000) sowie im Kontrollmedium wurden 2 Schalen mit jeweils 10 Jungfischen (also 20 Individuen) angesesetzt. Der Versuch begann um 11.00 Uhr morgens. In jeder Schale wurde bei 3 ruhenden Individuen die Kiemendeckelfrequenz bestimmt; alle angegebenen Mittelwerte beruhen daher jeweils auf 6 Einzelmessungen. Die in 1:4000, 1:8000 und 1:12000 sowie die im Kontrollmedium erhaltenen Mittelwerte und deren mittlere Abweichungen (senkrechte Linien) sind in Abbildung 4 dargestellt.

Die Kontrolltiere verändern ihre Kiemendeckelfrequenz während des 24stündigen Versuchs nur wenig. Dagegen erhöht sich die Frequenz unmittelbar nach Einbringung in die genannten Testmedien erheblich. In 1:4000 bleibt die erhöhte Frequenz bis kurz vor Eintritt des Todes erhalten; in 1:8000 und 1:12000 beginnt sie jedoch nach etwa 7 bis 9 Stunden wieder abzusinken und erreicht nach 21 Stunden in etwa den Kontrollwert. Diese Tendenz zur Normalisierung der Kiemendeckelfrequenz ist vermutlich zurïckzuführen auf (1) physiko-chemische Veränderungen in den "alternden" Testmedien und (2) Anpassungserscheinungen (nicht-genetische Adaptation) der Testorganismen.

Zwe ite Versuchsserie. Das Ergebnis der ersten Versuchsserie veranlaßte uns, die Kiemendeckelfrequenz auch in stärker verdünnten Testmedien und über eine mehr als doppelt so lange Zeitspanne zu untersuchen. In jedem Testmedium sowie im Kontrollmedium wurden 3 Schalen mit jeweils 10 Jungfischen (also 30 Individuen) 
angesetzt und in jeder Schale bei 3 ruhenden Individuen die Kiemendeckelfrequenz gemessen; alle angegebenen Mittelwerte beruhen daher jeweils auf 9 Einzelmessungen. Die Fische wurden bei Versuchsbeginn, nach 24 Stunden und nach 48 Stunden (jeweils gegen 11.00 Uhr morgens) in die 10 Minuten vorher angesetzten Medien umgesetzt. Während der Versuchsdauer betrugen die Ausfälle in 1:8000 23\%, 1:16000 10\%, $1: 240007 \%, 1: 320007 \%$ und im Kontrollmedium 3\%.

Wie bei der ersten Versuchsserie verändern die jungen Gobius pictus ihre Kiemendeckelbewegung im Kontrollmedium relativ wenig (Abb. 5). Einige Stunden nach dem Umsetzen kommt es jedoch regelmäßig zu einer gewissen Erhöhung der Frequenz. Da die Tiere bei künstlichem Dauerlicht gehalten wurden, offenbart sich hier möglicherweise eine endogene Tagesrhythmik.

Demgegenüber steigt die Kiemendeckelfrequenz nach dem ersten Umsetzen in die Testmedien steil an; erst später beginnt sie wieder abzufallen und sich dem Kontrollwert mehr oder minder anzunähern. Der gleiche Vorgang wiederholt sich im Prinzip nach dem zweiten und dritten Umsetzen. Das Ausmaß der Frequenzsteigerung läßt eine Beziehung zum Verdünnungsgrad erkennen: es erreicht Maximalwerte in 1:8000 und neigt dazu, mit zunehmender Verdünnung abzunehmen.

Leider mußte die Versuchsserie nach etwa 52 Stunden abgebrochen werden; es spricht jedoch manches dafür, daß die nach dem Umsetzen erreichten Gipfelwerte mit der Häufigkeit des Umsetzens abnehmen. Dieses Phänomen könnte als nicht-genetische Adaptation gedeutet werden. Langfristige Versuche mit normal ernährten Jungfischen müssen hier Aufschluß geben; unsere Versuchstiere wurden ja während der Versuche nicht gefüttert (p. 144).

\section{Experimente an Larven von Solea solea}

Als Versuchstiere dienten 6 Tage alte Larven (Totallänge 4,1 $\pm 0,2 \mathrm{~mm}$ ) der Seezunge Solea solea (Linnafus 1766). Ihre Eltern (Nordseetiere) hatten unter kontrollierten Laboratoriumsbedingungen gelaicht; Eientwicklung, Larvenschlupf und Aufwuchs erfolgten bei $20^{\circ} \mathrm{C}$ (FLüCHTER 1966). Unsere Untersuchungen wurden bei $200 \pm 1^{\circ} \mathrm{C}$ durchgeführt. Diese Temperatur liegt nach mündlicher Mitteilung von Herrn Dr. Fuüchter im Optimalbereich (Überlebens- und Wachstumsraten) für Seezungenlarven aus der Nordsee. Je 10 Larven wurden in runde Glasschalen (Durchmesser in Höhe der Wasseroberfläche: $124 \mathrm{~mm}$ ) mit jeweils $400 \mathrm{ml}$ Test- bzw. Kontrollmedium überführt (Abb. 1b). In jeder Verdünnungsstufe wurden 10 Tiere getestet. Im übrigen glichen die Versuchsbedingungen denen bei $G$. pictus.

Als Kriterium diente ausschließlich die Mortalitätsrate. Nach Aufhören jeglicher Lebensäußerungen (Kiemendeckelbewegungen, Reaktion auf Anstoßen) wurden die Tiere für tot erklärt und entfernt. Als Testmedien dienten die Verdünnungsstufen. 1:2500, 1:5000, 1:7500, 1:10000, 1:12500, 1:15000, 1:17500, 1:20000. Die Testzeit betrug 30 Stunden. Während dieser Zeit wurden die Larven nicht in neue Medien umgesetzt (Abb. 6).

In den Testmedien 1:2500, 1:5000, 1:7500 waren bereits nach 6 Stunden sämtliche Versuchstiere gestorben. In 1:10000 gingen während dieser Zeitspanne 9 Larven 


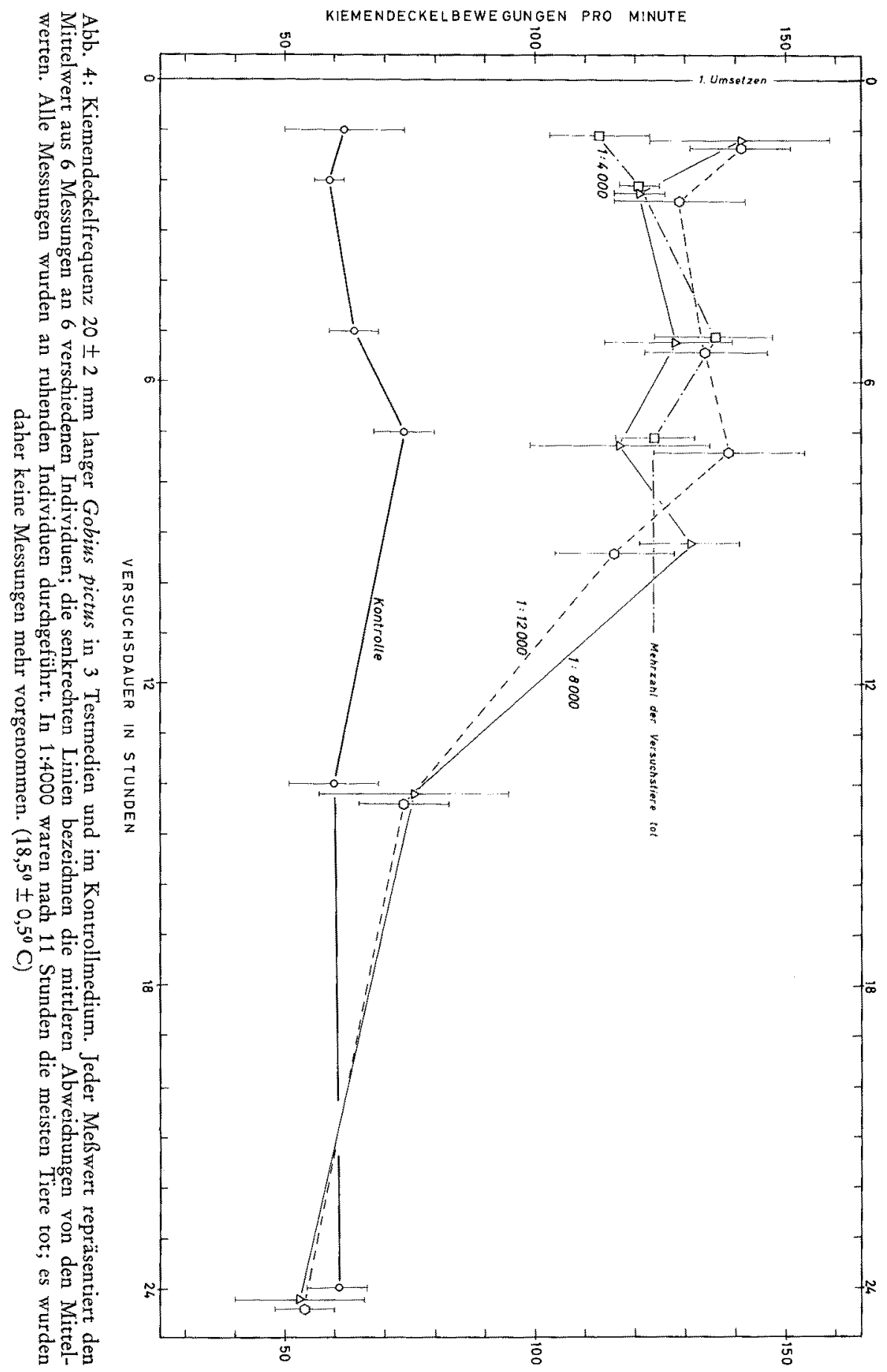




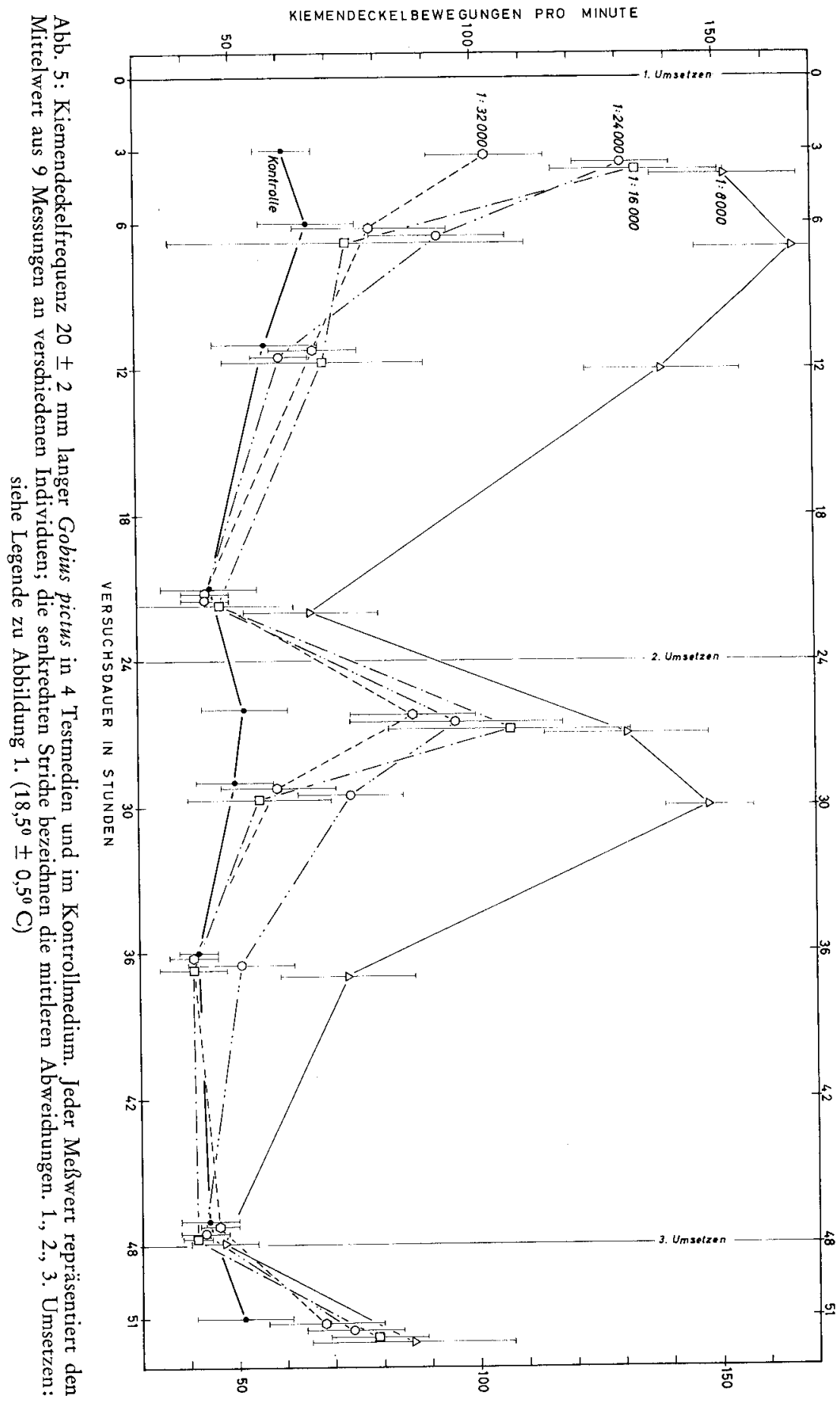




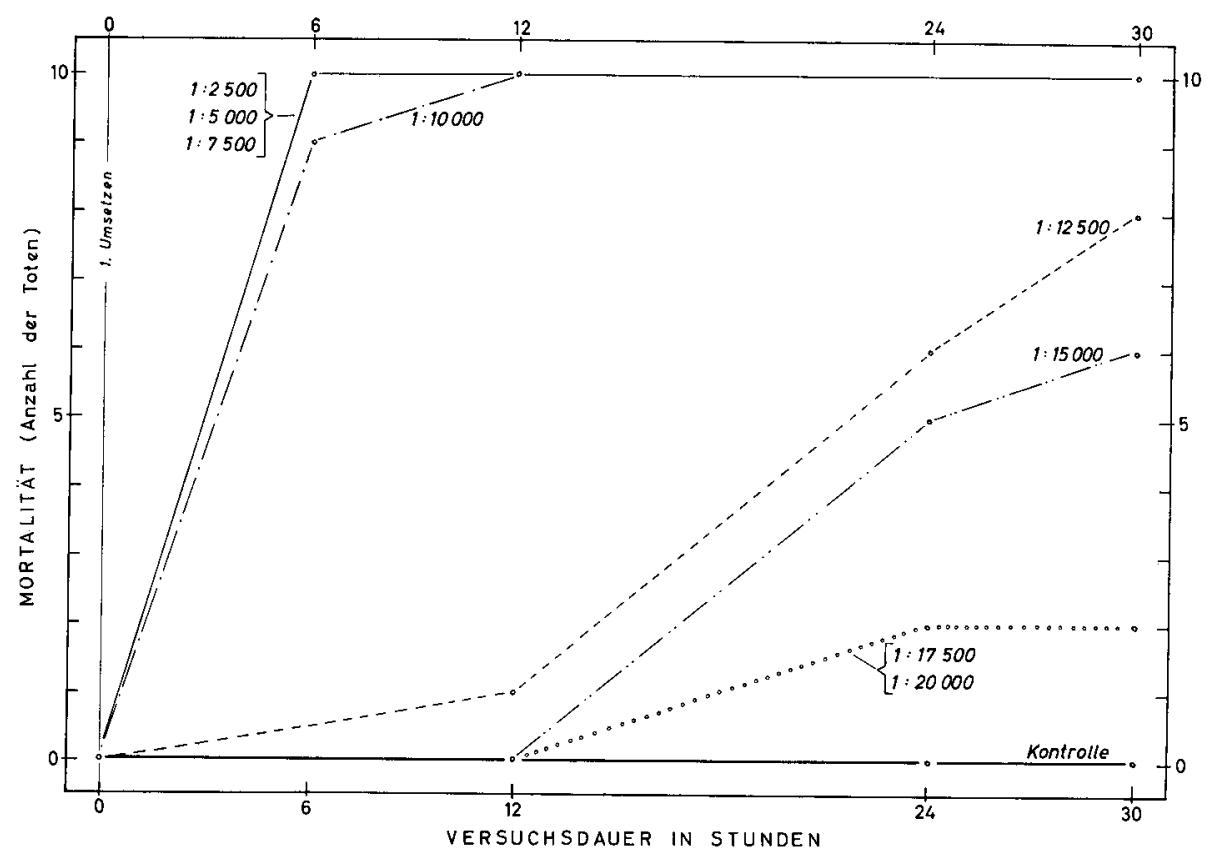

Abb. 6: Mortalität 6 Tage alter $(4,1 \pm 0,2 \mathrm{~mm}$ langer) Larven von Solea solea. In 8 Testmedien und im Kontrollmedium wurden jeweils je 10 Individuen getestet und die Anzahl der Toten registriert. $\left(20^{\circ} \pm 1^{\circ} \mathrm{C}\right)$

ein; die letzte verendete im Verlauf der nächsten 6 Stunden. In 1:12500 und 1:15000 starb die Hälfte der Larven oder mehr innerhalb von 24 Stunden. In 1:17500 und 1:20000 starben in der Zeit zwischen 12 und 24 Stunden nach Versuchsbeginn jeweils 2 Individuen. Im Kontrollmedium blieben während der 30stündigen Versuchszeit sämtliche Larven am Leben.

\section{DISKUSSION}

Unsere an den Jungtieren bzw. Larven der Nordseefische Gobius pictus und Solea solea erarbeiteten Versuchsergebnisse zeigen, daß die getesteten schwefelsäureund eisensulfathaltigen Industrieabwässer unter den geschilderten Versuchsbedingungen negative biologische Konsequenzen besitzen.

Bei den Jungtieren von Gobius pictus läßt sich eine deutlich erhöhte Mortalität in den Verdünnungsstufen 1:2000 bis 1:8000 nachweisen (Abb. 2, 3). Aber auch in $1: 12000,1: 16000,1: 24000$ und 1:32000 ist die Mortalität höher als bei den Kontrolltieren; zur statistischen Absicherung dieser Unterschiede sind weitere Untersuchungen mit umfangreicherem Tiermaterial erforderlich. Die 50\%-Letaldosis $\left(\mathrm{LD}_{50}\right)$ nach 24 Stunden liegt etwa bei 1:6000.

Die getesteten Larven von Solea solea sterben in 1:2500, 1:5000, 1:7500 und 
1:10000 innerhalb von 12 Stunden. Eine starke Erhöhung der Mortalität ist innerhalb von 30 Stunden noch bis zu Verdünnungen von 1:12500 und 1:15000 nachweisbar (Abb. 6). Auch in 1:17500 und 1:20000 ist die Mortalität höher als im Kontrollmedium; angesichts der geringen Anzahl an Versuchstieren muß die Signifikanz dieser Unterschiede durch weitere Untersuchungen mit umfangreicherem Larvenmaterial ermittelt werden. Die 50\%-Letaldosis nach 24 Stunden liegt etwa bei 1:15000. Die $S$. solea-Larven reagieren also empfindlicher auf die getesteten Abwässer als die Jungtiere von $G$. pictus.

Bei Jungfischen von Gobius pictus sind Veränderungen der Kiemendeckelfrequenz auch in stärker verdünnten Testmedien noch deutlich zu erkennen. Insbesondere kommt es kurz nach dem Umsetzen in neue Testmedien zu erheblichen, temporären Frequenzerhöhungen (Abb. 4, 5). Diese lassen sich noch bei einer Verdünnung von 1:32000 nachweisen. Sie sind von Verhaltensänderungen (erhöhte Unruhe) begleitet und werden als Reaktion auf vermehrten Umweltstress gedeutet.

Altere und größere Gobius pictus sind wesentlich resistenter als Jungfische. Dies geht aus unseren unpublizierten Vergleichsuntersuchungen an älteren Lebensstadien hervor. Leider konnten diese Vergleichsuntersuchungen nicht näher ausgewertet werden, weil sich das Aggressions- und Imponierverhalten subadulter und adulter Tiere, das vielfach zu Beißereien führte, als erheblicher Störfaktor erwies. Hier müssen weitere Versuche, bei denen den Einzelindividuen ein größeres Areal zur Verfügung steht, Aufschluß geben.

Auch bei einer Studie über die Reaktionen sich entwickelnder Embryonen und frisch geschlüpfter Larven des Herings (Clupea harengus) erwiesen sich schwefelsäureund eisensulfathaltige Industrieabwässer der gleichen Herkunft bis zu Verdünnungen von 1:32000 als gefährlich (KINNE \& Rosenthal 1967). Diese Untersuchungen waren - im Gegensatz zu den hier vorgelegten Ergebnissen - in dauerdurchlüfteten Medien durchgeführt worden. Die Zusammensetzung des Dünnsäure-Grünsalzgemisches wich geringfügig von dem hier benutzten Gemisch ab. In allen Testmedien (1:8000, $1: 16000,1: 24000,1: 32000$ ) reagierten 12 Tage alte Heringsembryonen kurz nach Versuchsbeginn mit einem temporären Anstieg der Herztätigkeit ("gealterte“ Testmedien; $8^{0} \pm 0,2^{\circ} \mathrm{C}$; etwa 16,5\% Salzgehalt). In Versuchen, in denen die gesamte Embryonalentwicklung (Befruchtung bis Schlüpfung) unter Einwirkung des Industrieabwassers $(1: 16000)$ stattfand, waren die Uberlebensrate und der prozentuale Schlupferfolg gegenüber den Kontrollen reduziert. Frisch geschlüptte, gesunde Larven wurden nach Umsetzen in 1:8000 weitgehend immobilisiert und starben innerhalb von 3 bis 6 Tagen; in 1:16000 war die Bewegungsaktivität und -koordination gestört; in 1:24000 und 1:32000 verhielten sich die Larven wie die Kontrolltiere, zeigten jedoch im Gegensatz zu diesen - keine Beutefanghandlungen.

Vorläufige Ergebnisse an Jungtieren von Gobius pictus in durchlüfteten Medien deuten darauf hin, daß die Mortalität geringfügig niedriger als im durchlüfteten Wasser ist. Die negativen biologischen Konsequenzen der Testmedien scheinen jedoch stärker von deren "Alter" (Zeitspanne nach dem Vermischen mit Meerwasser) abzuhängen als davon, ob sie durchliuftet werden oder nicht. Die maximale Giftigkeit wird offenbar zum Zeitpunkt der Vermischung erreicht; sie hält aber möglicherweise mehrere Stunden an. Auch Kinne \& Rosenthal (1967) haben bereits auf die Bedeutung des Alters 
der Testmedien hingewiesen. Der Einfluß des Alters der Testmedien auf das Ausmaß der biologischen Konsequenzen geht auch aus unseren bisher unpublizierten Versuchsergebnissen an 3 Wochen alten Larven des Steinpickers Agonus cataphractus hervor: Innerhalb von 12 Stunden verursachten 24 Stunden alte Testmedien eine 100\% \% ige Mortalität in 1:2500 und 1:5000; 4 Stunden alte Testmedien dagegen führten in der gleichen Zeitspanne noch in Verdünnungen von $1: 12500$ und $1: 15000 \mathrm{zu} 100 \%$ iger Mortalität (Sterblichkeit in den Kontrollmedien: $0 \%$. 13,5\% C; sonstige Versuchsbedingungen wie bei Solea solea).

Hinsichtlich der biologischen Konsequenzen der in Frage stehenden Industrieabwässer ist die Ansäurung des Meerwassers und die damit verbundene Erhöhung des $\mathrm{CO}_{2}$-Partialdrucks - bei gleichzeitiger Erniedrigung des $\mathrm{O}_{2}$-Partialdrucks durch die Oxydation von $\mathrm{Fe}^{\mathrm{II}}$ zu Fe $\mathrm{Fe}^{\mathrm{III}}$ - offensichtlich von größter Wichtigkeit. Auch die bei Verdünnung mit Meerwasser resultierenden Eisenhydroxid-Niederschläge können sich negativ auswirken. So kam es beispielsweise bei Larven des Einsiedlerkrebses Eupargurus bernhardus zu Anreicherungen der Niederschläge im Bereich der dorsalen Carapax-region sowie der Freß- und Schwimmfüße (unpublizierte eigene Ergebnisse). VeligerLarven der Mollusken Crepidula fornicata und Lacuna divaricata verfingen sich in den Ausflockungen und waren offensichtlich in ihrer Fortbewegung gehemmt (unpublizierte eigene Ergebnisse). Es muß damit gerechnet werden, daß diese Niederschläge Lokomotion, Nahrungsaufnahme und Respiration zahlreicher mariner Organismen beeinträchtigen können. Wo immer sie sedimentieren, drohen auch den auf oder im Sediment lebenden Organismen eine Reihe von Gefahren (Beeinflussung der Austauschvorgänge zwischen Sediment und freiem Wasser, Veränderung der Interstitialräume, Verklebungen an der Sedimentoberfläche).

Unsere in Glasschalen und Aquarien erhaltenen Laboratoriumsergebnisse lassen sich nicht ohne weiteres auf die Situation in der freien Nordsee extrapolieren. Sie er-" geben aber erste, wichtige Aufschlüsse über die Reaktionen der Versuchstiere auf die getesteten Verdünnungsstufen der in Frage stehenden Industrieabwässer unter defnierten Bedingungen.

\section{ZUSAMMENEASSUNG}

1. Die biologischen Konsequenzen schwefelsäure- und eisensulfathaltiger Industrieabwässer wurden unter einfachen, definierten Bedingungen im Laboratorium getestet. Es kamen Verdünnungen von 1:2000 bis 1:32000 zur Anwendung. Als Versuchstiere dienten Jungfische der Fleckengrundel Gobius pictus und Larven der Seezunge Solea solea, als Kriterien deren Mortalität (bei $G$. pictus außerdem die Kiemendeckelfrequenz).

2. Bei $20 \pm 2 \mathrm{~mm}$ langen Jungfischen von Gobius pictus läßt sich bei $18,5^{\circ} \mathrm{C}$ eine signifikant erhöhte Mortalität noch bei Verdünnungen bis zu 1:8000 nachweisen. Die 50\%-Letaldosis $\left(\mathrm{LD}_{50}\right)$ nach 24 Stunden liegt etwa bei 1:6000. Subletale Konsequenzen (starke, temporäre Intensivierung und Fluktuationserhöhung der Kiemendeckelfrequenz) sind noch bei Verdünnungen bis zu 1:32000 erkennbar. Sie werden als Indiz für erhöhten Umweltstress gedeutet. 
3. Sechs Tage alte Larven der Seezunge Solea solea (Totallänge: 4,1 $\pm 0,2 \mathrm{~mm}$ ) lassen bei $20^{\circ} \mathrm{C}$ noch bei Verdünnungen von $1: 17500 \mathrm{bzw}$. 1:20000 Erhöhung der Mortalität gegenüber den Kontrolltieren erkennen; ihre 50\%-Letaldosis nach 24 Stunden liegt etwa bei $1: 15000$. Sie reagieren empfindlicher als junge $G$. pictus,

4. Das Ausmaß der negativen biologischen Konsequenzen der getesteten Industrieabwässer nimmt zu mit der Konzentration des Testmediums. Es nimmt ab mit dem "Alter" des Testmediums (Zeitspanne zwischen Vermischen der Stammlösung mit Meerwasser und dem Beginn des Tests).

Herrn Dr. J. FLüchTER (Biologische Anstalt Helgoland, Meeresstation) danken wir herzlich für die freundliche Uberlassung des Seezungenmaterials, der Deutschen Forschungsgemeinschaft für finanzielle Unterstïtzung dieses Forschungsvorhabens (Ki 41/22).

\section{ZITIERTE LITERATUR}

FLüchter, J., 1966. Spawning, first feeding and larval behavior of the North Sea sole. In: C. M. (Council Meeting) International Council for the Exploration of the Sea (I.C.E.S.) Sect. C: Near Northern Seas Committee 3, 1-5.

Kinne, O. \& Rosenthal, H., 1967. Effects of sulfuric water pollutants on fertilization, embryonic development and larvae of the herring, Cimpea barengus. Mar. Biol, 1, 65-83.

\section{Diskussion im Anscbluß an den Vortrag Kinne \& Schumann}

HalsBand: Warum wurden die Versuche unter ortsfremden Voraussetzungen durchgeführt? Weder eine Durchlüftung noch Durchströmung der Versuchsgefäße wurde vorgenommen. Gerade bei Dünnsäure wird auch in geringen Konzentrationen, zumal die Versuchsgefäße relativ klein waren, ein $\mathrm{CO}_{2}$-Phänomen auftreten, wodurch die Versuchstiere eingehen müssen. Bei der Verklappung wird durch die starke Vermischung ein Vergleich mit diesen Laboratoriumsversuchen nicht möglich sein.

KINNE: Bei einem Vergleich mit der Situation im Meer stellen Versuche in 2 Liter fassenden Glasschalen stets „ortsfremde Voraussetzungen“ dar - gleichgültig, ob die Testmedien durchlüftet oder undurchlüftet sind. Wir haben uns bei diesen Untersuchungen für undurchlüftetes Wasser entschieden, weil es leichter durchschaubare Verhältnisse bietet. Orientierende Voruntersuchungen zeigten darüber hinaus, daß die Mortalitätsrate unter unseren Versuchsbedingungen stärker von Alter und Vorgeschichte der Versuchstiere abhängt als davon, ob die Medien durchlüftet oder undurchlüftet sind. Möglicherweise hängt dies mit der günstigen Oberflächen-Volumen-Relation der Medien in den von uns verwandten Glasschalen ab. $\mathrm{Ob}$ im übrigen durchlüftetes oder undurchlüftetes Wasser in unseren Versuchsgefäßen der Normalsituation im ausersehenen Verklappungsgebiet in der Nordsee näherkommt, läßt sich nicht ohne weiteres entscheiden. Der erste Teil Ihrer Aussage, daß „Gerade bei Dünnsäure ... auch in geringen Konzentrationen ... ein $\mathrm{CO}_{2}-\mathrm{Phänomen"} \mathrm{auftritt,} \mathrm{ist} \mathrm{bekannt} \mathrm{-} \mathrm{der} \mathrm{zweite:} \mathrm{"wo-}$ durch die Versuchstiere eingehen müssen" ist (in dieser Form und unter Anspielung auf die Nichtdurchlüftung), wie unsere Versuchsergebnisse zeigen, unrichtig.

$\mathrm{Daß}$ ein Vergleich unserer Laboratoriumsversuche mit der Situation in situ nicht ohne weiteres möglich ist, hat niemand bestritten. Im Gegenteil: Wir haben mehrfach darauf hingewiesen, daß ausreichend fundierte Vorhersagen bezuglich der im Meer zu erwartenden biologischen Konsequenzen erst möglich werden, wenn die in Aussicht genommenen langfristigen Untersuchungen über subletale Reaktionen (Wachstum, Nahrungsumsatz, Vermehrungsrate) 
und die Adaptationskapazität wichtiger Hauptvertreter aus dem betreffenden Meeresgebiet experimentell untersucht und Experimente in vereinfachten künstlichen Okosystemen (bestehend aus den Elementargliedern der Nahrungskette) angestellt worden sind. Nichtsdestoweniger weisen unsere ersten Untersuchungsergebnisse - die hier vorgetragenen ebenso wie die vorher von Krnne \& Rosenthal (Mar. Biol. 1, 65, 1967) mit Eiern und Larven des Herings Clupea barengus durchgeführten - darauf hin, daß die benutzten Testmedien nachweisbare negative biologische Konsequenzen (erhöhte Mortalität, Stress) besitzen. Es muß daher damit gerechnet werden, daß Abwasserkonzentrationen, die denen in unseren Versuchen benutzten entsprechen, auch im natürlichen Lebensraum eine Gefahr für vergleichbare Lebensstadien von Hering, Fleckengrundel und Seezunge darstellen.

\section{NACHTRAG}

Die Mischung von Dünnsäure und Grünsalz (p. 142) ist etwa 4 normal an $\mathrm{H}_{2} \mathrm{SO}_{4}$ und 4,3 normal an $\mathrm{FeSO}_{4}$. Da der Ansäurung des Meerwassers und besonders der dadurch verursachten Erhöhung des $\mathrm{CO}_{2}$-Partialdrucks biologisch vermutlich erhebliche

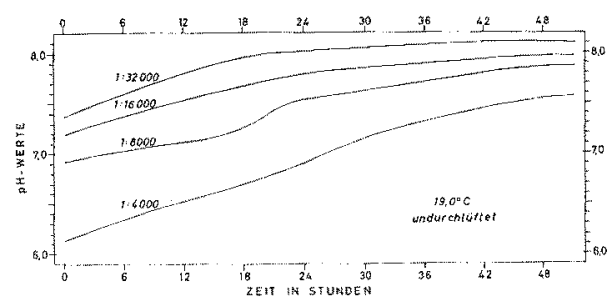

Abb. 7: Beispiel für die pH-Verschiebungen in undurchlïftetem Wasser (ohne Versuchstiere) als Funktion der Zeit $\left(19^{\circ} \mathrm{C}, 29,5\right.$ bis $30 \% 0 \mathrm{~S}$; sonstige Versuchsanordnung - Gefäß, Flüssigkeitsmenge - wie bei den Experimenten mit Gobius pictus). Die erste $\mathrm{pH}$ Messung erfolgte 2 bis 6 Minuten nach dem Herstellen der betreffenden Verdünnungsstufe

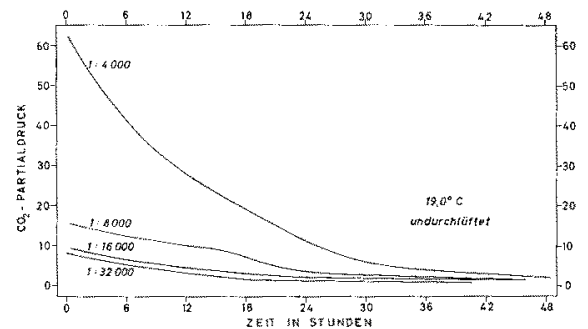

Abb. 9: Die anhand der pH-Kuryen in $\mathrm{Ab}$ bildung 7 rechnerisch ermittelten $\mathrm{CO}_{2}$-Kurven (COg-Partialdrud, bezogen auf Luf $=1$ )

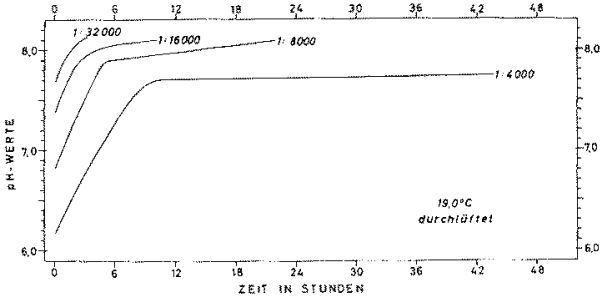

Abb. 8: Beispiel für die pH-Verschiebungen in durchlüftetem Wasser (ohne Versuchstiere) als Funktion der Zeit $\left(19^{\circ} \mathrm{C}, 29,5\right.$ bis $30 \% \mathrm{~S}$; sonstige Versuchsanordnung wie bei den Experimenten mit Gobius pictus). Die erste pH-Messung erfolgte 2 bis 6 Minuten nach dem Herstellen der betreffenden Verdünnungsstufe

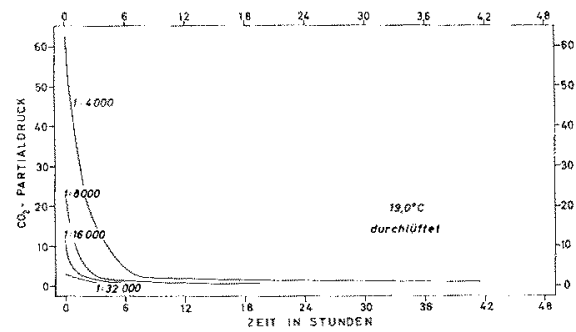

Abb. 10: Die anhand der pH-Kurven in $\mathrm{Ab}$ bildung 8 rechnerisch ermittelten $\mathrm{CO}_{2}-\mathrm{K}$ urven $\left(\mathrm{CO}_{2}\right.$-Partialdrud, bezogen auf Luf $\left.=1\right)$

Bedeutung zukommt, wurde der Verlauf der $\mathrm{pH}$-Verschiebungen von Herrn RosenTHAL experimentell verfolgt (Versuchsanordnung wie bei Gobius pictus; jedoch $19^{\circ} \mathrm{C}$, 29,5 bis $30 \%$ S). Beispiele der erhaltenen Kurven sind in Abbildung 7 für undurch- 
lüftetes Wasser und in Abbildung 8 für durchlüftetes $(110$ bis $145 \mathrm{ml}$ Luft pro Minute, handelsübliche Durchlüftungssteine) Wasser dargestellt. Die entsprechenden, von Herrn Dr. BoHLING errechneten $\mathrm{CO}_{2}$-Kurven geben Abbildungen 9 und 10 wieder. Kontrollanalysen haben ergeben, daß die vom Hersteller erhaltenen Ausgangsgemische hinsichtlich ihrer chemischen Zusammensetzung gewisse - meist geringfügige - Abweichungen aufweisen. Die hier publizierten Kurven können daher nur Annäherungswerte liefern. 